\title{
$\Rightarrow$ MEMORY
}

\section{A boost for medical marijuana?}

injections of
$\Delta^{9}$-THC in
mice caused
a sustained
increase in
hippocampal
levels of COX2
and its product
prostaglandin
E2

Marijuana is used to treat pain and other clinical conditions, but its use can lead to unwanted side effects, including cognitive impairment. A study in Cell now shows that marijuana use increases cyclooxygenase 2 (COX2; also known as PTGS2) signalling in the hippocampus and that this effect is linked to memory impairment, suggesting that COX2 inhibitors may enhance the medical utility of marijuana by reducing its side effects.

The main psychoactive ingredient of marijuana is $\Delta^{9}$-tetrahydrocannabinol ( $\Delta^{9}$-THC), and Chen et al. set out to identify the mechanisms by which it impairs memory function. They focused on COX2 as a potential mediator because the endocannabinoid 2-arachidonylglycerol (2-AG) has been shown to inhibit COX2 signalling. The authors were therefore surprised to find that repeated intraperitoneal injections of $\Delta^{9}$-THC in mice caused a sustained increase in hippocampal levels of COX2 and its product prostaglandin E2 (PGE2). Administration of a COX2 inhibitor prevented the $\Delta^{9}$-THC-induced increase in PGE2 levels. Moreover, they showed that the contrasting effects of $\Delta^{9}$-THC and 2-AG on COX2 activity both involve the $\mathrm{G}_{\mathrm{i} / \mathrm{o}}$ protein-coupled cannabinoid 1 receptor (CB1R) but that different $G$ protein subunits mediate these opposing effects: specifically, G $\beta \gamma$ subunits mediate the effect of $\Delta^{9}$-THC, and the $\mathrm{Ga}_{\mathrm{i}}$ subunit that of 2-AG.
As shown previously, daily injections with $\Delta^{9}$-THC reduced both hippocampal long-term potentiation and performance on hippocampusdependent memory tasks in mice. These effects were prevented by concurrent administration of a COX2 inhibitor and did not occur in transgenic mice lacking COX $2 . \Delta^{9}$-THC injections also caused morphological changes in the hippocampus that have been associated with such impairments in synaptic plasticity and memory, including a reduced density of dendritic mushroom spines containing AMPA and NMDA receptors in CA1 neurons. Moreover, $\Delta^{9}$-THC decreased the expression of the postsynaptic scaffolding protein PSD95 (also known as DLG4) and the expression of several synaptic and extrasynaptic glutamate receptor subunits. Administration of a COX2 inhibitor prevented all of these effects.

To determine whether administration of a COX2 inhibitor interferes with any of the medically beneficial effects of marijuana, the authors examined a transgenic mouse model of Alzheimer's disease. They found that daily $\Delta^{9}$-THC injections for 4 weeks reduced levels of both amyloid- $\beta$ and neurodegeneration (by increasing levels of the enzyme neprilysin, which can degrade amyloid- $\beta$ ), regardless of whether the injections were accompanied by administration of a COX2 inhibitor.
This suggests that COX2 inhibition does not affect this particular beneficial effect of $\Delta^{9}$-THC.

Together, these findings point to the possibility that COX2 inhibitors, including widely available nonsteroidal anti-inflammatory drugs such as ibuprofen, could be used to prevent at least some of the unwanted side-effects of the marijuana component $\Delta^{9}$-THC without impairing its beneficial properties.

Leonie Welberg

ORIGINAL RESEARCH PAPER Chen, R. et al. $\triangle^{9}$-THC-caused synaptic and memory impairments are mediated through COX-2 signaling. Cell 155 , 1154-1165 (2013) 